\title{
OPEN Critical current modulation induced by an electric field in superconducting tungsten-carbon nanowires
}

\author{
Pablo Orús ${ }^{1,2}$, Vladimir M. Fomin ${ }^{3,4,5}$, José María De Teresa ${ }^{1,2,6 \bowtie}$ \& Rosa Córdoba ${ }^{7 凶}$
}

The critical current of a superconducting nanostructure can be suppressed by applying an electric field in its vicinity. This phenomenon is investigated throughout the fabrication and electrical characterization of superconducting tungsten-carbon (W-C) nanostructures grown by $\mathrm{Ga}^{+}$focused ion beam induced deposition (FIBID). In a $45 \mathrm{~nm}$-wide, $2.7 \mu \mathrm{m}$-long W-C nanowire, an increasing side-gate voltage is found to progressively reduce the critical current of the device, down to a full suppression of the superconducting state below its critical temperature. This modulation is accounted for by the squeezing of the superconducting current by the electric field within a theoretical model based on the Ginzburg-Landau theory, in agreement with experimental data. Compared to electron beam lithography or sputtering, the single-step FIBID approach provides with enhanced patterning flexibility and yields nanodevices with figures of merit comparable to those retrieved in other superconducting materials, including $\mathrm{Ti}$, $\mathrm{Nb}$, and $\mathrm{Al}$. Exhibiting a higher critical temperature than most of other superconductors, in which this phenomenon has been observed, as well as a reduced critical value of the gate voltage required to fully suppress superconductivity, W-C deposits are strong candidates for the fabrication of nanodevices based on the electric field-induced superconductivity modulation.

In the semiconductor industry, the term field effect is used to define the modulation of the charge carrier density that takes place in the active channel of a semiconductor when an electric field is externally applied ${ }^{1}$. This electric field-induced resistivity control is at the core of the operation of field-effect transistors (FETs). FETs are employed as active electronic components that exploit the field effect for electrical signal switching and amplification ${ }^{2}$. Particularly, metal oxide semiconductor FETs (MOSFETs) fabricated on integrated circuits represent the most extensively used building block in that industry for the fabrication of a wide range of electronic-based devices thanks to their simplicity and affordability ${ }^{3}$.

The field effect is virtually non-existent in metallic materials, since the large density of charge carriers present in a metal promptly results in the screening of externally applied electric fields over very short distances. However, recent calculations indicate that such a field is able to extend within a superconducting material over at least the value of its coherence length ${ }^{4}$, and the study of the phenomenon in superconducting materials has gained interest in recent years due to its potential application in superconducting electronics ${ }^{5}$.

Central to this interest is the fact that an increasing electric field applied in close proximity to a superconducting bridge has been recently found to progressively suppress the critical current of superconducting channels. This electric field-induced quenching of the superconducting state has been observed in different superconducting materials, mostly purely metallic: in titanium transistor-like devices, both in-plane ${ }^{6}$ and suspended ${ }^{7}$; in aluminium nanodevices with a single back-gate ${ }^{8}$ or side-gate ${ }^{9}$, deducing from the latter a weak coupling

${ }^{1}$ Instituto de Nanociencia y Materiales de Aragón (INMA), CSIC-Universidad de Zaragoza, 50009 Zaragoza, Spain. ${ }^{2}$ Departamento de Física de la Materia Condensada, Facultad de Ciencias, Universidad de Zaragoza, 50009 Zaragoza, Spain. ${ }^{3}$ Institute for Integrative Nanosciences (IIN), Leibniz Institute for Solid State and Material Research (IFW) Dresden, Helmholtzstraße 20, 01069 Dresden, Germany. ${ }^{4}$ Laboratory of Physics and Engineering of Nanomaterials, Department of Theoretical Physics, Moldova State University, Strada A. Mateevici 60, 2009 Chişinău, Republic of Moldova. ${ }^{5}$ Institute of Engineering Physics for Biomedicine, National Research Nuclear University MEPhl, Kashirskoe shosse 31, Moscow 115409, Russia. ' Laboratorio de Microscopías Avanzadas (LMA), University of Zaragoza, 50018 Zaragoza, Spain. ${ }^{7}$ Instituto de Ciencia Molecular (ICMol), Universitat de València, 46980 Paterna, Spain. ${ }^{\circledR}$ email: deteresa@unizar.es; rosa.cordoba@uv.es 
between the electric field and an external magnetic field; in vanadium Dayem nano-bridges, operated as electrical rectifiers ${ }^{10}$; and in niobium gate-controlled transistors, also exploited as half-wave rectifiers ${ }^{11}$. Uniquely, and contrarily to other works, an enhancement of the critical current with increasing gate voltage has also been observed in niobium nitride thin micro- and nano-bridges, being ascribed to changes in the superconducting vortex surface barrier ${ }^{12}$.

The microscopical justification for the occurrence of this effect, which cannot be justified in the Barden-Cooper-Schrieffer (BCS) framework, is not fully accounted for at the present time and is the subject of an unsettled debate ${ }^{13-15}$, with separate intrinsic and extrinsic effects potentially contributing in various geometries and in different superconducting materials. Ritter et al. ${ }^{16}$ ascribe the critical current quenching observed in titanium nitride nanowires to the injection of energetic electrons from the gate electrodes to the superconductor, which trigger the formation of a large number of quasiparticles that drive the nanowire back to the normal state. Alegria et al. ${ }^{17}$ and Golokolenov et al. ${ }^{18}$ support similar arguments to account for the behavior observed in electron tunneling spectroscopy experiments in titanium nanowires and in a vanadium waveguide resonator, respectively. On the other hand, Mercaldo et al. ${ }^{19}$ propose a theoretical model based on electric-field induced spin-orbit polarization at the surface, capable of modulating the phase and amplitude of the superconducting order parameter. Similarly, Chirolli et al. ${ }^{20}$ present a model for crystalline superconductors, in which the effect is accounted for by a local modification of the density of states of the material induced through Rashba-like surface effects. Solinas et al. ${ }^{21}$ describe a Sauter-Schwinger effect in BCS superconductors to account for the phenomenon.

The empirical observation of the superconducting current quenching proves a highly promising path towards the fabrication of superconducting FET (S-FET) nanodevices, which can simultaneously benefit from the nondissipating regime characteristic to superconducting materials, and from the electric switching capability of devices controlled by the application of a gate voltage $\mathrm{e}^{22,23}$. Additional prospective applications include the exploitation of the effect in spin-filter Josephson junctions ${ }^{24}$, logic gates ${ }^{25}$, and single-photon detectors ${ }^{26}$.

The precise nanofabrication of such devices can be achieved by means of the focused ion beam induced deposition (FIBID) procedure. FIBID is a direct-write nanopatterning technique that makes use of a focused beam of energetic $\mathrm{Ga}^{+}$ions (FIB) to locally induce the decomposition of a gaseous precursor material previously adsorbed on the surface of the substrate. With a spot size of $5 \mathrm{~nm}$, as the beam scans the surface of the substrate, the gaseous monolayer of precursor is decomposed at and near the irradiated area following the pattern traced by the beam, resulting in the growth of a pattern-shaped nanodeposit ${ }^{27,28}$. A prime example of the capabilities of this technique is the recently reported fabrication of $\mathrm{NbC}$ nanodevices for vortex dynamic investigation via FIBID of a Nb-based gaseous precursor ${ }^{29}$.

FIBID of the $\mathrm{W}(\mathrm{CO})_{6}$ precursor results in the growth of a W-C based material with an atomic content of $\mathrm{W}$ of 40-50\%, a nanocrystallite-based microstructure, and a room-temperature resistivity ( $\rho 300 \mathrm{~K})$ of $200-300 \mu \Omega$ $\mathrm{cm}$. Paramountly, the material is type-II superconductor, with a critical temperature $\left(T_{c}\right)$ around $4.75 \mathrm{~K}$, an upper critical magnetic field of 9.5 T, a critical current density of $0.01-0.10 \mathrm{MA} / \mathrm{cm}^{2}$, and values for the coherence length and penetration depth of $6.25 \mathrm{~nm}$ and $850 \mathrm{~nm}$, respectively ${ }^{30-35}$. Superconductivity has also been found to occur in the material yielded when the decomposition of $\mathrm{W}(\mathrm{CO})_{6}$ is induced by a focused electron beam instead, which has been exploited for the fabrication of Josephson junctions ${ }^{36}$. Remarkably, and contrary to most of the aforementioned superconducting materials, in which this phenomenon has been investigated, $\mathrm{Ga}^{+}$FIBID W-C is not fully metallic, nor it is a highly crystalline, yet it allows for the occurrence of the effect, as it will be presented in the following sections. The novel exploitation the $\mathrm{Ga}^{+}$FIBID technique for the direct nanopatterning of W-C S-FET devices further settles it as a solid technique for the fabrication of superconducting components in advanced nanodevices.

The patterning flexibility and the single-step nature of the FIBID procedure make for highly convenient clean growth and handling of a superconducting material. As such, $\mathrm{Ga}^{+}$FIBID of $\mathrm{W}-\mathrm{C}$ is a strong approach for the further investigation of electric field-induced superconductivity modulation. The experimental observation of such a phenomenon in $\mathrm{Ga}^{+}$FIBID W-C nanowires is presented here. A full suppression of the superconducting state below the critical temperature of the material is achieved at a remarkably low side-gate voltage of $3 \mathrm{~V}$. A theoretical model based on the Ginzburg-Landau (GL) theory is provided, which appropriately reproduces the experiments. The transconductance of the nanodevice is comparable by value to that retrieved in the aforementioned works in other superconducting materials, further fueling the potential of FIBID of W(CO) ${ }_{6}$ as a solid technique for the fabrication of S-FET devices.

\section{Methods}

$\mathrm{Si} / \mathrm{SiO}_{2}$ pieces were used as substrates. A supporting $\mathrm{Cr} / \mathrm{Au}$ structure consisting of current/voltage and gate electrodes was defined by electron beam lithography (EBL) in a Raith Group PIONEER Two EBL/Scanning Electron Microscope (SEM) instrument, and grown in an electron-beam evaporator, eliminating the EBL resist by liftoff.

The two gate contacts were initially patterned as a continuous, $45 \mathrm{~nm}$-thick film. A $200 \mathrm{~nm}$-wide, $2.7 \mu \mathrm{m}$-long gap was milled between the two selectively removing material using the $\mathrm{Ga}^{+} \mathrm{FIB}^{37}$ exposing the underlying $\mathrm{Si}$ / $\mathrm{SiO}_{2}$ substrate. The milling was carried out using the same operating parameters employed in growth (without the injection of the precursor material) and in the same instrument, both described below.

FIBID and scanning electron microscopy (SEM) imaging of the samples were carried out in a commercial Thermo Fisher Helios 600 NanoLab FIB/SEM microscope, equipped with a $\mathrm{Ga}^{+}$column with a liquid metal ion source. In the FIBID procedure, an acceleration voltage of $30 \mathrm{kV}$ and an ion beam current of $1.8 \mathrm{pA}$ were used. The process chamber had a base pressure of $10^{-6} \mathrm{mbar}$, which was raised to $10^{-5}$ mbar during the injection of the gaseous $\mathrm{W}(\mathrm{CO})_{6}$ precursor. During growth, the nozzle used to deliver the precursor was positioned $50 \mu \mathrm{m}$ away from the irradiation point in the vertical direction, and $100 \mu \mathrm{m}$ away in the in-plane direction. 


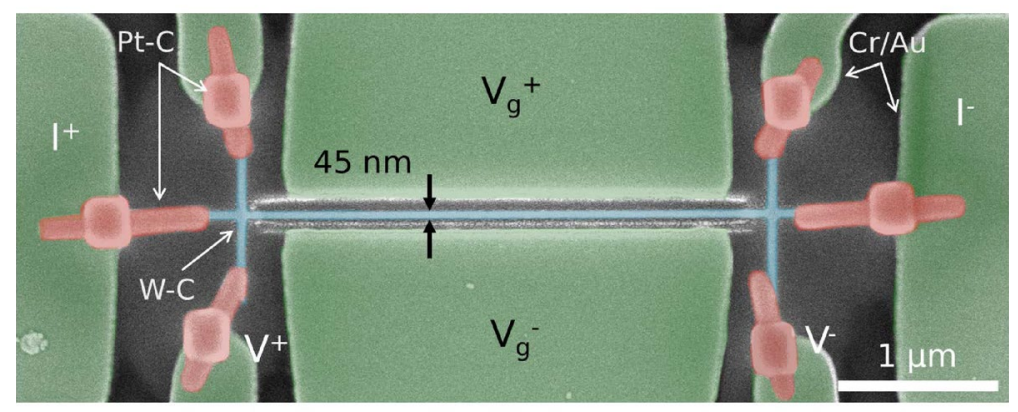

(a) Artificially colored SEM image of a representative sample. The $\mathrm{Cr} / \mathrm{Au}$ pads patterned by EBL are shown in green, and the FIBID-grown PtC contacts and W-C nanowire are shown in red and blue, respectively.

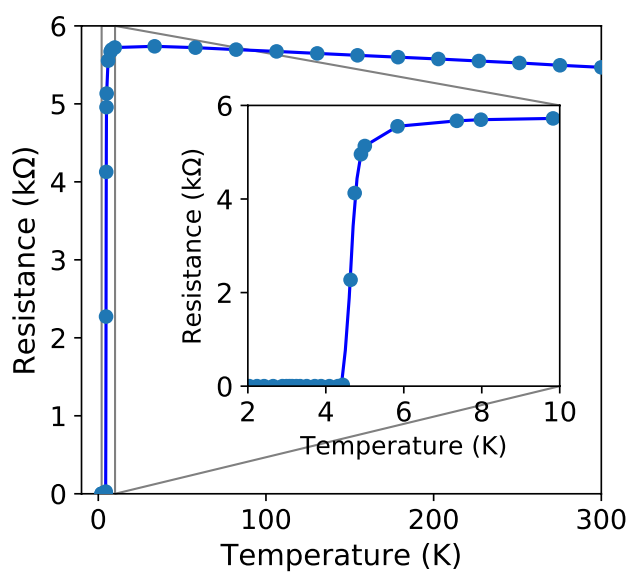

(b) Temperature dependence. Inset: zoomed view of the 2-10 K region.

Figure 1. SEM image and superconducting transition of a nanodevice. In (b), solid lines are a guide for the eye.

The deposits were patterned as straight, narrow nanowires with two (equally wide) perpendicular leads crossing at each end, with length between leads of $3.3 \mu \mathrm{m}$, width of $45 \mathrm{~nm}$ (nominally $20 \mathrm{~nm}$ ), and a nominal thickness of $30 \mathrm{~nm}$ (Fig. 1a). The used values of volume per dose, dwell time, and overlap were to $8.2 \cdot 10^{-2} \mu \mathrm{m}^{3} / \mathrm{nC}, 500$ $\mu s$, and $50 \%$, respectively. The total deposition time for each deposit was 15 seconds. Growing the deposit in the $200 \mathrm{~nm}$-wide gap yielded in a nanowire-electrode separation of around $75 \mathrm{~nm}$ at each side.

Lastly, the ends of the nanowire were joined to the metallic pads by growing supporting electrical contacts via FIBID of the $\left(\mathrm{CH}_{3}\right)_{3}\left(\mathrm{CH}_{3} \mathrm{CpPt}\right)$ precursor, known to present a metallic content high enough to be used for this purpose $^{38,39}$. They were grown as $150 \mathrm{~nm}$-wide, $100 \mathrm{~nm}$-thick leads with a supporting $300 \times 300 \mathrm{~nm}, 100 \mathrm{~nm}$-thick square grown at the joining point with the pads. In this FIBID procedure, the ion beam current, acceleration voltage, dwell time, and overlap were set to $1.8 \mathrm{pA}, 30 \mathrm{kV}, 200 \mathrm{~ns}$, and $0 \%$, respectively. The volume per dose in this growth was $0.5 \mu \mathrm{m}^{3} / \mathrm{nC}$.

The device was designed in this manner for the driving current to be injected along the long axis of the nanowire while the voltage drop was measured in the $3.3 \mu \mathrm{m}$-long channel between the leads, in a four probe measurement fashion. The gate voltage was applied using the two large pads located in close proximity to this channel.

The low-temperature electrical characterization of the samples was performed in a commercial Quantum Design Physical Property Measurement System instrument. Measurements were taken down to $2 \mathrm{~K}$. Electrical contact between the instrument and the sample was achieved by ultrasonic wire-bonding of aluminum wires of the EBL-patterned metallic contacts to the instrument sampleholder.

\section{Results and discussion}

The experimental results strengthen the applicability of $\mathrm{Ga}^{+}$FIBID of $\mathrm{W}(\mathrm{CO})_{6}$ for the fabrication of S-FET nanodevices. Superconductivity in $\mathrm{W}-\mathrm{C}$ nanowires patterned using this technique is experimentally found to be progressively suppressed with increasing values of gate voltage, up to a full transition to the normal state for a gate voltage of around $3 \mathrm{~V}$ at $2 \mathrm{~K}$.

Experimental results. The nanowires exhibited an estimated room-temperature resistivity of $230 \mu \Omega \cdot \mathrm{cm}$ and a temperature-induced transition to the superconducting state in the vicinity of $T_{c}=4.75 \mathrm{~K}$ (defining $T_{c}$ as the temperature at which the resistance takes half its value in the normal state, at $10 \mathrm{~K}$ ) (Fig. 1a). Contrary to $\mathrm{Al}, \mathrm{Ti}$, and $\mathrm{Nb}$, and similarly to the $\mathrm{NbN}$ material, $\mathrm{Ga}^{+}$FIBID $\mathrm{W}-\mathrm{C}$ presents a non-metallic response above its critical temperature, with a residual-resistivity ratio at $10 \mathrm{~K}\left(\rho_{300 \mathrm{~K}} / \rho_{10 \mathrm{~K}}\right)$ of 0.96 .

The modulation of the critical current induced by the electric field was assessed by measuring current-resistance curves of the nanowire below the critical temperature while sequentially increasing the voltage difference between the gate electrodes, thus obtaining the resistance as a function of the driving current (Fig. 2). To account for possible hysteresis effects, each measurement consisted of a complete cycle, increasing the driving current from 0 to $\pm 3 \mu \mathrm{A}$, and then decreasing it again down to zero.

The critical current of the device (here defined as the value of the driving current at which the resistance of the device reaches $90 \%$ of its non-superconducting, normal value) monotonically decreased with increasing gate voltage. In the absence of gate voltage, at $2 \mathrm{~K}$ a critical current density of $0.24 \mathrm{MA} / \mathrm{cm}^{2}$ is detected. The depairing current density ${ }^{40,41}$ takes an estimated value of $2.37 \mathrm{MA} / \mathrm{cm}^{2}$. The obtained values of critical current density are comparable to those obtained in different devices of W-C grown via FIBID, with both $2 \mathrm{D}^{35}$ and $3 \mathrm{D}^{42} \mathrm{Ga}^{+}$and $\mathrm{He}^{+}$W-C FIBID nanostructures, respectively. A comparable critical current density of $0.18 \mathrm{MA} / \mathrm{cm}^{2}$ is reported in similar Ti nanodevices ${ }^{43}$.

At $2 \mathrm{~K}$, a complete suppression of the superconducting state for applied gate voltages of around $3 \mathrm{~V}$ (Fig. 2a) was observed. The response of the device is asymmetric with respect to the sign of the current: for positive values 


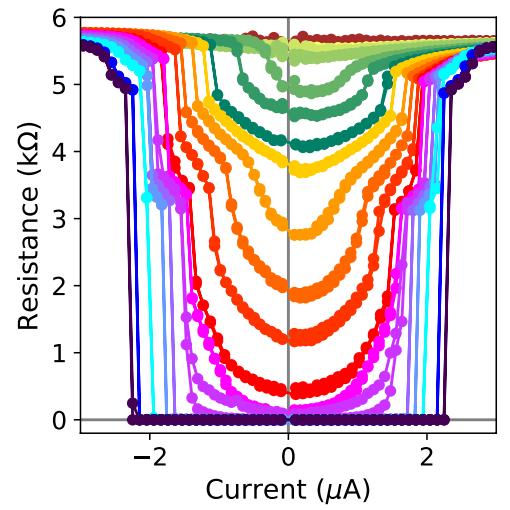

(a) $2 \mathrm{~K}$

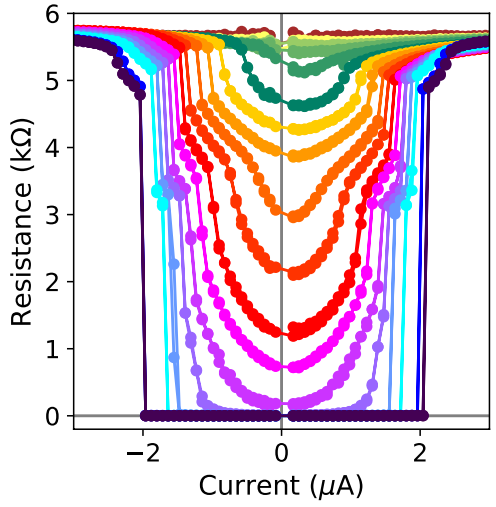

(b) $3 \mathrm{~K}$.

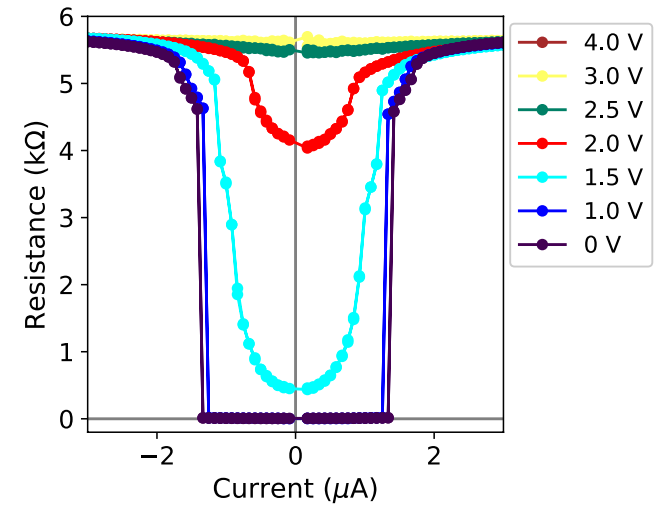

(c) $4 \mathrm{~K}$.

Figure 2. Electric field-induced critical current modulation at 2, 3, and $4 \mathrm{~K}$ under gate voltages from 0 to $4 \mathrm{~V}$. Solid lines are a guide for the eye. In (a) and (b) all unlabeled curves (between 1.5 and 3.0 V) are 0.1 V apart.

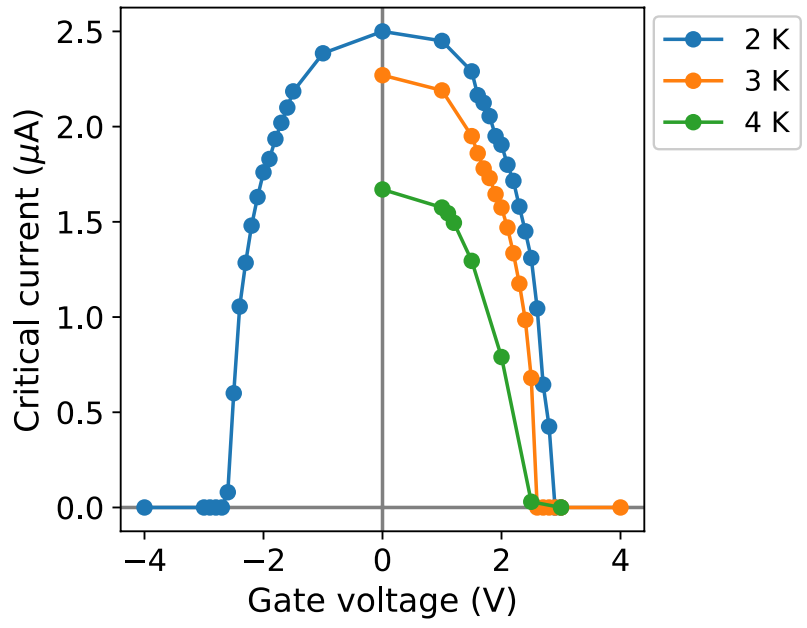

Figure 3. Critical current as a function of the gate voltage. Solid lines are a guide for the eye.

of the gate voltage, the onset of the modulation is favored at negative values of the driving current. This effect cannot be fully accounted for at the present time, although it is not unreasonable for it to be caused by leakage current effects flowing to and from the gate contacts ${ }^{15}$.

No hysteresis was detected in the devices, indicating that no significant thermal heating effects take place in the nanowires at the experiment conditions. Similar behavior was observed at temperatures of 3 and $4 \mathrm{~K}$ (Fig. 2b,c, respectively).

Some resistance-current curves exhibited a transition to the normal state in the form of two independent steps, pointing towards the local appearance of non-superconducting areas in the superconducting channel that yield a non-zero resistance as the current flows through. These localized normal regions appear by the electric field-induced squeezing of the superconducting current, which gains relevance as the gate voltage and the temperature increase. The squeezing eventually leads to the whole nanowire being forced into the normal state below its critical temperature (further discussion on this matter follows in the next section).

Similarly, finite resistance values were detected at very low values of driving current for gate voltage values above $2 \mathrm{~V}$ at $2 \mathrm{~K}$. At 3 and $4 \mathrm{~K}$, as the critical current of the device decreased with increasing temperature, the extent of the electric field-induced modulation was also shifted towards lower values of the current (Fig. 3).

Between gate voltages of 2 and $3 \mathrm{~V}$, at very low values of driving current the nanowire is already in a resistive state (yet with an absolute value of resistance lower than that in the normal state), also in agreement with the proposed interpretation in terms of the squeezing effect.

Theoretical model. De Simoni et al. ${ }^{43}$ proposed an ad hoc theoretical model based on the GL theory to describe the experimentally observed modulation of the critical current in titanium nano-bridges as a function of temperature and gate voltage. A description which is presented here, is also built in the GL framework, providing with added robustness by including the boundary conditions.

In a superconducting wire affected by an external electric field, the superconducting current experiences a squeezing effect by the electric field perpendicular to the wire. Therefore, to develop a minimal model of the 
critical current in a superconducting wire, the two key conceptual ingredients are the superconducting current flowing along the wire, and the compression of the superconducting state across the wire.

The proposed model of the critical current in a superconducting wire of length $L$ (along the $x$-axis) and a rectangle-shaped cross-section sized $w_{y} \times w_{z}$ (along the $y$ - and $z$-axes, respectively), is based on the GL free energy functional $F^{40}$ :

$$
F=\xi^{2}|\nabla \psi|^{2}-|\psi|^{2}+\frac{1}{2 \psi_{0}^{2}}|\psi|^{4}+F_{n},
$$

where $F_{n}$ is the free energy of the normal state and other fields, $\xi$ is the coherence length, and $\psi$ is the order parameter, with $\psi_{0}$ being the value it takes infinitely deep in the bulk of the superconductor. It is supplemented with the boundary conditions on the edges ${ }^{40}$, both with the surrounding insulator:

$$
\left.\nabla_{y} \psi\right|_{y= \pm w_{y} / 2}=0,\left.\quad \nabla_{z} \psi\right|_{z= \pm w_{z} / 2}=0
$$

and with the contacts. As a simplification, the latter are selected in the form of equality of the superconducting current $j^{40}$ and the transport current $j_{t r}$ densities at the contacts:

$$
j=\left.\frac{e^{*}}{m^{*}}|\psi|^{2} \hbar \nabla_{x}(\operatorname{Arg} \psi)\right|_{\text {contact }} \equiv j_{t r},
$$

where $e^{*}$ and $m^{*}$ are the effective charge and mass, respectively.

The basic conceptual ingredients of the model are as follows: (i) The order parameter in the superconducting regions carrying the superconducting current along the $x$-axis can be selected in the form of a plane wave,

$$
\psi=e^{i k x} \psi_{1}(y, z)
$$

with the parameter $k$ determining the superconducting current (shown below). (ii) For the effect of the external electric field, the order parameter can be used in the form of the wave function of a ground state of a harmonic oscillator normalized within the wire ${ }^{43}$ :

$$
\begin{aligned}
& \psi_{\sigma_{y}}(y)=\sqrt{w_{y}} \frac{\exp \left(-\frac{y^{2}}{4 \sigma_{y}^{2}}\right)}{\sqrt{\sqrt{2 \pi} \sigma_{y}}}, \\
& \psi_{\sigma_{z}}(z)=\sqrt{w_{z}} \frac{\exp \left(-\frac{z^{2}}{4 \sigma_{z}^{2}}\right)}{\sqrt{\sqrt{2 \pi} \sigma_{z}}},
\end{aligned}
$$

where the dispersion values $\sigma_{y}$ and $\sigma_{z}$ (along the $y$ - and $z$-axes, respectively) are inversely proportional to the corresponding components of the electric field ${ }^{43}: 1 / \sigma_{y}=E_{y} / V_{y}$ and $1 / \sigma_{z}=E_{z} / V_{z}$. A significant compression of the area of superconductivity towards the center of the cross-section implies $\sigma_{y} \ll w_{y}$ and $\sigma_{z} \ll w_{z}$, leading to an exponentially strong decay towards the edges, so that the boundary conditions (Eq. 3 ) are approximately satisfied with high accuracy.

Thus, the order parameter can be selected in the form of Eq. (4) with the Ansatz $\psi_{1}(y, z)=\psi_{2} \psi_{\sigma_{y}}(y) \psi_{\sigma_{z}}(z)$ to retrieve the superconducting current as

$$
I(k)=\int_{-w_{y} / 2}^{w_{y} / 2} \int_{-w_{z} / 2}^{w_{z} / 2} j d y d z=\frac{\psi_{2}^{2} \hbar e^{*}}{m^{*}} w_{y} w_{z} k .
$$

A variation of the functional $F$ (Eq. 1) with respect to $\psi_{1}$ yields the GL equation:

$$
\xi^{2}\left[k^{2}+\left(\frac{y}{2 \sigma_{y}^{2}}\right)^{2}+\left(\frac{z}{2 \sigma_{z}^{2}}\right)^{2}\right] \psi_{1}-\psi_{1}+\frac{1}{\psi_{0}^{2}} \psi_{1}^{3}=0 .
$$

Averaging Eq. (6) over the cross-section of the wire yields $\psi_{2}$ and subsequently the superconducting current $I$ (Eq. 5) as a cubic function of $k$ :

$$
I(k)=\frac{\psi_{0}^{2} \hbar e^{*}}{m^{*}} w_{y} w_{z}\left(k\left[1-\frac{\xi^{2}}{48}\left(\frac{w^{2}}{\sigma_{y}^{4}}+\frac{v^{2}}{\sigma_{z}^{4}}\right)\right]-\xi^{2} k^{3}\right) .
$$

A condition for the current to be maximal is a vanishing derivative of $I(k)$, which results in:

$$
k^{2}=\frac{1}{3 \xi^{2}}\left[1-\frac{\xi^{2}}{48}\left(\frac{w_{y}^{2}}{\sigma_{y}^{4}}+\frac{w_{z}^{2}}{\sigma_{z}^{4}}\right)\right]
$$

and the critical current follows as 


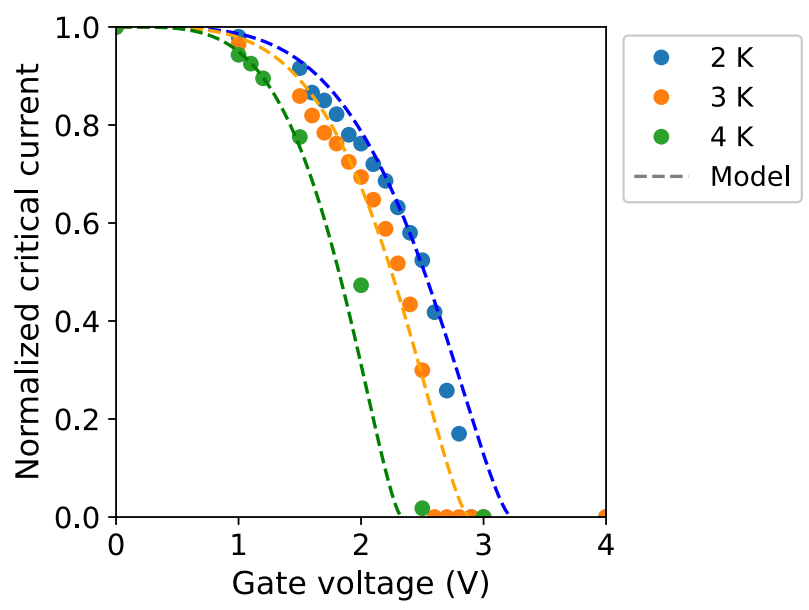

Figure 4. Normalized critical current-gate voltage characteristic. Dots indicate the experimental data at 2,3, and $4 \mathrm{~K}$. Dashed lines represent the predicted characteristics at the corresponding temperatures (Eq. 10) with $V_{g}^{c}=3.7 \mathrm{~V}$

$$
I_{c} \equiv \max I(k)=\frac{2 \psi_{0}^{2} \hbar e^{*}}{3 \sqrt{3} m^{*} \xi} w_{y} w_{z}\left[1-\frac{\xi^{2}}{48}\left(\frac{w_{y}^{2}}{\sigma_{y}^{4}}+\frac{w_{z}^{2}}{\sigma_{z}^{4}}\right)\right] .
$$

Using the explicit dependence on temperature,

$$
\psi_{0}^{2}(T)=\psi_{0}^{2}(0)\left(1-\frac{T}{T_{c}}\right), \quad \xi(T)=\xi(0)\left(1-\frac{T}{T_{c}}\right)^{-1 / 2},
$$

we arrive at

$$
I_{c}=\frac{2 \psi_{0}^{2}(0) \hbar e^{*}}{3 \sqrt{3} m^{*} \xi(0)} w_{y} w_{z}\left(1-\frac{T}{T_{c}}\right)^{3 / 2}\left[1-\left(1-\frac{T}{T_{c}}\right)^{-1}\left(\frac{w_{y}^{2}}{\sigma_{y}^{4}}+\frac{w_{z}^{2}}{\sigma_{z}^{4}}\right)\right]^{3 / 2} .
$$

If the electric field is applied in the $y$-direction, Eq. (9) results in:

$$
I_{c}(T, E)=I_{c}(0,0)\left(1-\frac{T}{T_{c}}\right)^{3 / 2}\left[1-\left(1-\frac{T}{T_{c}}\right)^{-1}\left(\frac{E}{E_{c}}\right)^{4}\right]^{3 / 2},
$$

where $E_{c}$ (and correspondingly, $V_{g}^{c}=E_{c} w_{y}$ ) represents the critical value of the electric field (gate voltage), at which superconductivity is fully suppressed at zero temperature, and

$$
I_{c}(0,0)=\frac{2 \psi_{0}^{2}(0) \hbar e^{*}}{3 \sqrt{3} m^{*} \xi(0)} w_{y} w_{z}, \quad \frac{1}{E_{c}^{4}}=\frac{\xi^{2}(0) w_{y}^{2}}{48 V_{y}^{4}} .
$$

The critical current as a function of the gate voltage (Eq. 10) is in good agreement with the experimental data (Fig. 4). Importantly, the critical value of the gate voltage, at which superconductivity is fully suppressed, decreases with increasing temperature.

Comparison to other materials. The performance of S-FET devices is typically assessed by means of the transconductance, a figure of merit which quantifies the variation in the current with the applied gate voltage, and is calculated here as the numerical derivative of the $I_{c}\left(V_{g}\right)$ experimental curves, $g_{m}=d I_{c} / d V_{g}$. The absolute values and temperature trend of the transconductance in the $\mathrm{W}-\mathrm{C}$ nanodevices (Fig. 5 ) are both comparable to those of other S-FET devices made of other superconducting materials, with the notable difference of a reduced value of critical gate voltage (Table 1 ).

The response of $\mathrm{W}-\mathrm{C}$ nanostructures to the electric field does occur in the reduced temperature interval $T / T_{c}=(0.421,0.842)$ (between 2 and $\left.4 \mathrm{~K}\right)$, and most importantly, a full suppression of the superconducting state is achieved at the low voltage of $V_{g}^{c} \sim 3 \mathrm{~V}$ at $T / T_{c}=0.421(2 \mathrm{~K})$, one order of magnitude below the majority of the reported values for other superconductor materials, with the lowest figure (observed in Ti nanostructures) still exceeding by 2.6 times the value retrieved in $\mathrm{W}-\mathrm{C}$. The associated values of reduced temperature at which the maximum values of transconductance are detected are on par with those reported in vanadium nanodevices ${ }^{8}$, and above most of the other studied materials. The absolute value of $T_{c}$ in $\mathrm{Ga}^{+}$FIBID provides a nanodevice operating temperature range wider than most of the other reported materials, surpassed only by $\mathrm{Nb}$ and competitive with $\mathrm{V}$. 


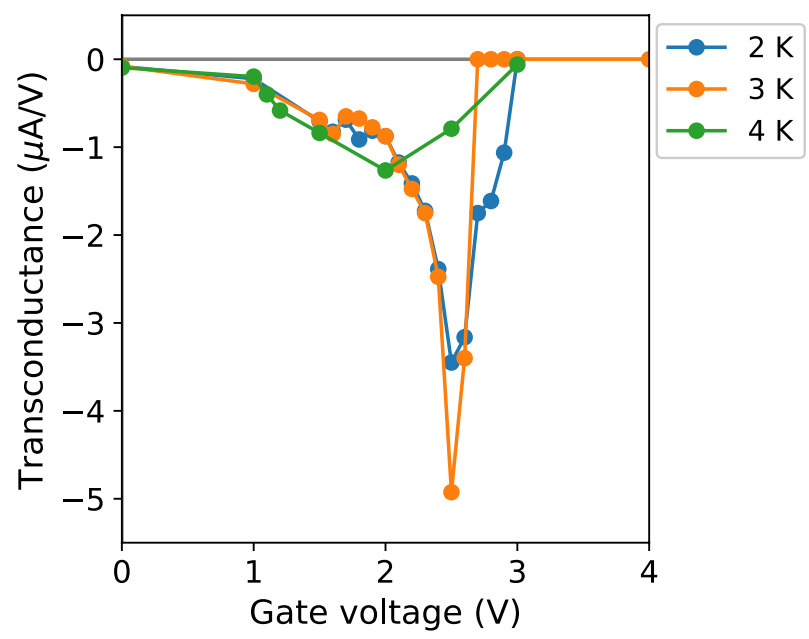

Figure 5. Transconductance. Solid lines are a guide for the eye.

\begin{tabular}{|l|l|l|l|}
\hline & $\mathbf{T}_{\mathbf{c}}(\mathbf{K})$ & $\mathbf{V}_{\mathbf{g}}^{\mathbf{c}}(\mathbf{V})$ & $\mathbf{g}_{\mathbf{m}}(\mu \mathbf{A} / \mathbf{V})$ \\
\hline $\mathrm{W}-\mathrm{C}$ & 4.75 & $3(0.421)$ & $5(0.631)$ \\
\hline $\mathrm{Ti}^{6}$ & 0.54 & $32 / 8(0.093)$ & $15(0.278)$ \\
\hline $\mathrm{V}^{8}$ & 5 & $13(0.675)$ & $150(0.575)$ \\
\hline $\mathrm{Al}^{9}$ & 0.60 & $25(0.083)$ & - \\
\hline $\mathrm{Nb}^{11}$ & 7.90 & $>40(\gtrsim 0.253)$ & $1.6(0.004)$ \\
\hline
\end{tabular}

Table 1. Maximum values of the relevant parameters in S-FET nanodevices. Quantities in parentheses indicate the reduced temperature $T / T_{c}$ at which the corresponding parameter was retrieved.

Moreover, FIBID is a direct nanopatterning technique, requiring a single step to grow nanostructures patterned at will, offering great flexibility in pattern design and shape fidelity without requiring the use of resists, solvents, etc. that might compromise the final quality of the nanostructures.

\section{Conclusions}

The modulation of the critical current by an electric field has been experimentally observed in superconducting $\mathrm{W}$-C nanostructures for the first time. The single-step $\mathrm{Ga}^{+}$FIBID procedure used to fabricate these nanodevices does not require the usage of resists, and presents great flexibility in the way nanostructures are patterned, providing with great freedom in pattern design and realization. As such, the experimental finding of a significant field-induced critical current modulation in W-C settles the FIBID technique as relevant and convenient for the nanofabrication framework of S-FET nanodevices.

Quantitatively, results retrieved in the W-C nanostructures are comparable to those obtained in similar vanadium-based nanodevices ${ }^{6}$, with a remarkably low value of the critical gate voltage, required to fully quench the superconducting state, of $3 \mathrm{~V}$ at a reduced temperature $T / T_{c}=0.421$. This gate voltage value is comparable to those used in MOSFET technology, and highly competitive in comparison to those reported in other superconducting materials.

A theoretical model based on the Ginzburg-Landau theory is proposed here, appropriately explaining the experimental data. Accounting for the effect of the squeezing of the supercurrent by the electric field, it adds further insight to the puzzling origin of the phenomenon, which has been investigated in different geometries and other materials, and is subjected to controversy at the present time $7,9,16,19,20$.

Nanoelectronics based on the electric field-induced modulation of the superconducting state are bound to see an inspiring development in the coming years. Functional nanodevices built upon this principle, such as gate-controlled $\mathrm{NbN}$ nanowires operating with coherent quantum phase slips ${ }^{44}$, and $\mathrm{Ti}$ and $\mathrm{V}$ rectifiers ${ }^{15}$ have already been realized. The scope of applications in this framework is wide, with the possibility of fabricating qubits, Josepshon junctions, logic gates, and single-photon detectors.

Received: 14 June 2021; Accepted: 18 August 2021

Published online: 06 September 2021

\section{References}

1. Nishizawa, J.-I. Junction field-effect devices. In Semiconductor Devices for Power Conditioning 241-272 (Springer, 1982). https:// doi.org/10.1007/978-1-4684-7263-9_11 
2. Dacey, G. \& Ross, I. The field effect transistor. Bell Syst. Tech. J. 34, 1149-1189. https://doi.org/10.1002/j.1538-7305.1955.tb037 94.x (1955).

3. Liou, J. J., Ortiz-Conde, A. \& Garcia-Sanchez, F. MOSFET Physics and Modeling 1-108 (Springer, 1998).

4. Ummarino, G. A. et al. Proximity Eliashberg theory of electrostatic field-effect doping in superconducting films. Phys. Rev. B 96, 064509. https://doi.org/10.1103/PhysRevB.96.064509 (2017).

5. IEE. International roadmap for devices and systems, cryogenic electronics and quantum information processing (2020 Edition).

6. Paolucci, F., De Simoni, G., Strambini, E., Solinas, P. \& Giazotto, F. Ultra-efficient superconducting Dayem bridge field-effect transistor. Nano Lett. 18, 4195-4199. https://doi.org/10.1021/acs.nanolett.8b01010 (2018).

7. Rocci, M. et al. Gate-controlled suspended titanium nanobridge supercurrent transistor. ACS Nano 14, 12621-12628. https://doi. org/10.1021/acsnano.0c05355 (2020).

8. Paolucci, F. et al. Field-effect control of metallic superconducting systems. AVS Quantum Sci. 1, 016501. https://doi.org/10.1116/1. 5129364 (2019).

9. Bours, L., Mercaldo, M., Cuoco, M., Strambini, E. \& Giazotto, F. Unveiling mechanisms of electric field effects on superconductors by a magnetic field response. Phys. Rev. Res. 2, 033353. https://doi.org/10.1103/PhysRevResearch.2.033353 (2020).

10. Puglia, C., De Simoni, G., Ligato, N. \& Giazotto, F. Vanadium gate-controlled Josephson half-wave nanorectifier. Appl. Phys. Lett. 116, 252601. https://doi.org/10.1063/5.0013512 (2020).

11. De Simoni, G., Puglia, C. \& Giazotto, F. Niobium Dayem nano-bridge Josephson gate-controlled transistors. Appl. Phys. Lett. 116, 242601. https://doi.org/10.1063/5.0011304 (2020).

12. Rocci, M. et al. Large enhancement of critical current in superconducting devices by gate voltage. Nano Lett. 21, 216-221. https:// doi.org/10.1021/acs.nanolett.0c03547 (2021).

13. Virtanen, P., Braggio, A. \& Giazotto, F. Superconducting size effect in thin films under electric field: Mean-field self-consistent model. Phys. Rev. B 100, 224506. https://doi.org/10.1103/PhysRevB.100.224506 (2019).

14. Puglia, C., De Simoni, G., \& Giazotto, F. (2021). Phase slips dynamics in gated Ti and V all-metallicsupercurrent nano-transistors: a review. arXiv preprint arXiv:2102.10699.

15. Puglia, C., De Simoni, G. \& Giazotto, F. Gate control of superconductivity in mesoscopic all-metallic devices. Materials 14, 1243. https://doi.org/10.3390/ma14051243 (2021).

16. Ritter, M. et al. A superconducting switch actuated by injection of high-energy electrons. Nat. Commun. 12, 1266. https://doi.org/ 10.1038/s41467-021-21231-2 (2021).

17. Alegria, L. D. et al. High-energy quasiparticle injection into mesoscopic superconductors. Nat. Nanotechnol. 16, 404-408. https:// doi.org/10.1038/s41565-020-00834-8 (2021).

18. Golokolenov, I., Guthrie, A., Kafanov, S., Pashkin, Y. A. \& Tsepelin, V. On the origin of the controversial electrostatic field effect in superconductors. Nat. Commun. 12, 1-7 (2021).

19. Mercaldo, M. T., Solinas, P., Giazotto, F. \& Cuoco, M. Electrically tunable superconductivity through surface orbital polarization. Phys. Rev. Appl. 14, 034041. https://doi.org/10.1103/PhysRevApplied.14.034041 (2020).

20. Chirolli, L., Cea, T. \& Giazotto, F. Impact of electrostatic fields in layered crystalline BCS superconductors. Phys. Rev. Res. 3, 023135. https://doi.org/10.1103/PhysRevResearch.3.023135 (2021).

21. Solinas, P., Amoretti, A. \& Giazotto, F. Sauter-Schwinger effect in a Bardeen-Cooper-Schrieffer superconductor. Phys. Rev. Lett. 126, 117001. https://doi.org/10.1103/PhysRevLett.126.117001 (2021).

22. Paolucci, F. et al. Magnetotransport experiments on fully metallic superconducting Dayem-bridge field-effect transistors. Phys. Rev. Appl. 11, 024061. https://doi.org/10.1103/PhysRevApplied.11.024061 (2019).

23. Paolucci, F. et al. Field-effect controllable metallic Josephson interferometer. Nano Lett. 19, 6263-6269. https://doi.org/10.1021/ acs.nanolett.9b02369 (2019).

24. De Simoni, G., Strambini, E., Moodera, J. S., Bergeret, F. S. \& Giazotto, F. Toward the absolute spin-valve effect in superconducting tunnel junctions. Nano Lett. 18, 6369-6374. https://doi.org/10.1021/acs.nanolett.8b02723 (2018).

25. Lee, S.-B., Hutchinson, G. D., Williams, D. A., Hasko, D. G. \& Ahmed, H. Superconducting nanotransistor based digital logic gates. Nanotechnology 14, 188. https://doi.org/10.1088/0957-4484/14/2/317 (2003).

26. Kadin, A. \& Johnson, M. Nonequilibrium photon-induced hotspot: A new mechanism for photodetection in ultrathin metallic films. Appl. Phys. Lett. 69, 3938-3940. https://doi.org/10.1063/1.117576 (1996).

27. Utke, I., Hoffmann, P. \& Melngailis, J. Gas-assisted focused electron beam and ion beam processing and fabrication. J. Vacuum Sci. Technol. B 26, 1197-1276. https://doi.org/10.1116/1.2955728 (2008).

28. Orús, P., Córdoba, R. \& De Teresa, J. Focused ion beam induced processing. In De Teresa, J. (ed.) Nanofabrication: Nanolithography Techniques and Their Applications (IOP, 2020) https://doi.org/10.1088/978-0-7503-2608-7ch5.

29. Dobrovolskiy, O. et al. Ultra-fast vortex motion in a direct-write Nb-C superconductor. Nat. Commun. 11, 1-10 (2020).

30. Sadki, E. H. S., Ooi, S. \& Hirata, K. Focused-ion-beam-induced deposition of superconducting nanowires. Appl. Phys. Lett. 85, 6206-6208. https://doi.org/10.1063/1.1842367 (2004).

31. Guillamón, I. et al. Nanoscale superconducting properties of amorphous W-based deposits grown with a focused-ion-beam. $N$. J. Phys. 10, 093005. https://doi.org/10.1088/1367-2630/10/9/093005 (2008).

32. Guillamón, I. et al. Direct observation of melting in a two-dimensional superconducting vortex lattice. Nat. Phys. 5, 651-655. https://doi.org/10.1038/nphys1368 (2009).

33. Li, W., Fenton, J. C., Gu, C. \& Warburton, P. A. Superconductivity of ultra-fine tungsten nanowires grown by focused-ion-beam direct-writing. Microelectron. Eng. 88, 2636-2638. https://doi.org/10.1016/j.mee.2010.12.116 (2011).

34. Córdoba, R. et al. Magnetic field-induced dissipation-free state in superconducting nanostructures. Nat. Commun. 4, 1437. https:// doi.org/10.1038/ncomms2437 (2013).

35. Córdoba, R. et al. Long-range vortex transfer in superconducting nanowires. Sci. Rep. 9, 12386. https://doi.org/10.1038/s41598019-48887-7 (2019)

36. Blom, T. J. et al. Direct-write printing of Josephson junctions in a scanning electron microscope. ACS Nano 15, 322-329 (2020).

37. Gierak, J. Focused ion beam nano-patterning from traditional applications to single ion implantation perspectives. Nanofabrication 1, 35-53. https://doi.org/10.2478/nanofab-2014-0004 (2014).

38. De Teresa, J. et al. Origin of the difference in the resistivity of as-grown focused-ion-and focused-electron-beam-induced Pt nanodeposits. J. Nanomater. 2009, 936863. https://doi.org/10.1155/2009/936863 (2009).

39. Fernández-Pacheco, A., De Teresa, J., Córdoba, R. \& Ibarra, M. Metal-insulator transition in Pt-C nanowires grown by focusedion-beam-induced deposition. Phys. Rev. B 79, 174204. https://doi.org/10.1103/PhysRevB.79.174204 (2009).

40. Tinkham, M. Introduction to Superconductivity (Courier Corporation, 2004).

41. Dinner, R. B., Robinson, A. P., Wimbush, S. C., MacManus-Driscoll, J. L. \& Blamire, M. G. Depairing critical current achieved in superconducting thin films with through-thickness arrays of artificial pinning centers. Supercond. Sci. Technol. 24, 055017 (2011).

42. Córdoba, R. et al. Three-dimensional superconducting nanohelices grown by He+-focused-ion-beam direct writing. Nano Lett. 19, 8597-8604 https://doi.org/10.1021/acs.nanolett.9b03153(2019).

43. De Simoni, G., Paolucci, F., Solinas, P., Strambini, E. \& Giazotto, F. Metallic supercurrent field-effect transistor. Nat. Nanotechnol. 13, 802-805. https://doi.org/10.1038/s41565-018-0190-3 (2018).

44. Anwar, M. S., \& Fenton, J. C. (2021). Gate-controlled conductance of superconducting NbN nanowires: coherent quantum phaseslips or Coulomb blockade? arXiv preprint arXiv:2104.09331. 


\title{
Acknowledgements
}

P.O. acknowledges Aragón Government for funding. The project that gave rise to these results received the support of a fellowship from "la Caixa" Foundation (ID 100010434). The fellowship code is LCF/BQ/PR19/11700008. Funding from the the European Union's Horizon 2020 research and innovation programme under the grant agreement No 892427 has been received. Authors acknowledge financial support from the Spanish Ministry of Economy and Competitiveness through Projects MAT2018-102627-T and MAT2017-82970-C2-2-R, from CSIC through project PIE202060E187, and from the Aragón Regional Government (Construyendo Europa desde Aragón) through Project E13_20R, with European Social Fund funding. The microscopy works have been conducted in the Laboratory for Advanced Microscopies (LMA), at the Institute of Nanoscience and Materials of Aragón (INMA) - University of Zaragoza. Authors acknowledge the LMA for offering access to their instruments and expertise. Authors acknowledge the use of the Physical Measurements Service in Servicio General de Apoyo a la Investigación (SAI)-University of Zaragoza. This work has been supported by projects H2020 (FATMOLSproject) and Excellence Unit María de Maeztu (CEX2019-000919-M). This work has been supported by the German Research Foundation (DFG) project \#FO 956/6-1 (Germany) and European Cooperation in Science and Technology-COST Action \#CA16218 (NANOCOHYBRI). V.M.F. acknowledges partial support from the MEPhI (Russia).

\section{Author contributions}

R.C. conceived the project. R.C. and J.M.D.T. supervised the experiments. P.O. and R.C. carried out the experiments. P.O. performed the experimental analysis. V.M.F. performed the theoretical analysis. P.O. and V.M.F. wrote the first version of the manuscript, and all authors contributed to its final form.

\section{Competing interests}

The authors declare no competing interests.

\section{Additional information}

Correspondence and requests for materials should be addressed to J.M.D.T. or R.C.

Reprints and permissions information is available at www.nature.com/reprints.

Publisher's note Springer Nature remains neutral with regard to jurisdictional claims in published maps and institutional affiliations.

\begin{abstract}
(c) (i) Open Access This article is licensed under a Creative Commons Attribution 4.0 International cc) License, which permits use, sharing, adaptation, distribution and reproduction in any medium or format, as long as you give appropriate credit to the original author(s) and the source, provide a link to the Creative Commons licence, and indicate if changes were made. The images or other third party material in this article are included in the article's Creative Commons licence, unless indicated otherwise in a credit line to the material. If material is not included in the article's Creative Commons licence and your intended use is not permitted by statutory regulation or exceeds the permitted use, you will need to obtain permission directly from the copyright holder. To view a copy of this licence, visit http://creativecommons.org/licenses/by/4.0/.
\end{abstract}

(C) The Author(s) 2021 